\title{
Correspondence on "Significance of automated external defibrillator in identifying lethal ventricular arrhythmias"
}

\author{
GuanYang Kang ${ }^{1}$ \\ Received: 30 August 2019 / Revised: 30 August 2019 / Accepted: 20 September 2019 /Published online: 22 October 2019 \\ (C) Springer-Verlag GmbH Germany, part of Springer Nature 2019
}

To the Editor:

We read with great interest the extremely informative paper recently published by Tsuda et al. [2] demonstrating that $16 \%$ of patients are initially managed for possible seizure disorders until automated external defibrillator (AED) recording identified lethal ventricular arrhythmia. We completely agree with their landmark conclusions. Their study can provide valuable lessons for the public access defibrillation program in China.

Recently, a study presented by Zhang and colleagues reported that "AEDs were installed in the specialized cabinet and sited inside the buildings with their availability only during the working hours. $80 \%$ AED cabinets were locked with keys. Of these, $25.7 \%$ were with keys obtainable from staff, $11.5 \%$ with keys nearby the cabinets, and $62.8 \%$ with no information about how to get the keys. All locked AEDs were equipped with a hammer to break the box in case of an emergency" [3]. We are surprised that so many AEDs were kept in locked cabinets in China. Can you imagine trying to save a person in cardiac arrest by running to the nearest lifesaving AED to find it in a locked cabinet, where you have to open it with a key or break it with a hammer? Optimal conditions for defibrillation are present for only $1-2$ min with success rates decreasing thereafter by at least $10 \%$ per minute of delay [1]. The quicker an AED is attached to a patient in cardiac arrest, the quicker a shock can be given (if indicated) to treat the arrhythmia causing the cardiac arrest. Locked cabinets inevitably introduce a delay in obtaining a defibrillator

This is a correspondence to the article https://doi.org/10.1007/s00431019-03421-9

Communicated by Peter de Winter

GuanYang Kang

gykang2008@126.com

1 Department of Cardiology, The Fifth People's Hospital of Dongguan, The Dongguan Affiliated Hospital of Medical College of Jinan University (also called Taiping People's Hospital of Dongguan), 111, Humen Road, Humen Dongguan 523000 Guangdong China and applying it to a person in cardiac arrest. Given the importance of reducing to a minimum the time taken to administer a shock, we believe that no delays or constraints should be placed on any person willing to use an AED nor should there be any physical barrier to restrict the immediate use of an AED such as a locked cabinet.

Author Contributions The author is solely responsible for the content of this manuscript.

\section{Compliance with ethical standards}

Conflict of interest The author declares that there is no conflict of interest.

\section{References}

1. Cummins RO, Ornato JP, Thies WH, Pepe PE (1991) Improving survival from sudden cardiac arrest: the "chain of survival" concept. A statement for health professionals from the advanced cardiac life support subcommittee and the emergency cardiac care committee, American Heart Association. Circulation 83:1832-1847

2. Tsuda T, Geary EM, Temple J (2019) Significance of automated external defibrillator in identifying lethal ventricular arrhythmias. Eur J Pediatr 178(9):1333-1342

3. Zhang L, Li B, Zhao X et al (2019) Public access of automated external defibrillators in a metropolitan city of China. Resuscitation 140:120-126

Publisher's note Springer Nature remains neutral with regard to jurisdictional claims in published maps and institutional affiliations. 